\title{
A Fuzzy Segmentation Method for Images of Heat-Emitting Objects
}

\author{
Anna Fabijańska \\ Department of Computer Engineering, Technical University of Lodz \\ 18/22 Stefanowskiego Str., 90-924, Lodz, Poland \\ an_fab@kis.p.lodz.pl
}

\begin{abstract}
In this paper a problem of soft image segmentation is considered. An approach for segmenting images of heat-emitting specimens is introduced. Proposed algorithm is an extension of fuzzy C-means (FCM) clustering method. Results of applying the algorithm to exemplary images of heat-emitting specimens are presented and discussed. Moreover the comparison with results of standard fuzzy C-means clustering is provided.
\end{abstract}

Keywords: image segmentation, fuzzy sets, clustering methods, FCM, hightemperature measurement, surface property of metal.

\section{Introduction}

Image segmentation is an essential task in machine vision applications. It addresses the problem of partitioning the image into disjoint regions according to the specific features (gray levels, texture etc.) [1]. Different approaches to image segmentation have been proposed in the literature. The most popular are histogram-based methods [1][2], region growing approaches [1][3], edge-based methods [1][4], clustering techniques [5] and watershed segmentation [1][6]. However most of these methods are hard techniques which provide crisp segmentation of images by qualifying each pixel to the unique region.

Hard segmentation techniques are often insufficient for practical applications of vision systems. Crisp partitioning of the image is often inaccurate and erroneous. Therefore the growth of interest in soft segmentation techniques can be observed recently. They are based on fuzzy set theory [7][8] end extract fuzzy regions (subsets of pixels) from the fuzzy image. In soft segmentation approaches each pixel can be qualified into multiple regions with different degree of membership [7][9][10].

\section{The Experimental Set Up}

Images considered in this paper were obtained from computerized system for hightemperature measurements of surface properties of metals and alloys. The system "Thermo-Wet" determines wetting angles and surface tension of liquid materials up to $1800^{\circ} \mathrm{C}$. The description of "Thermo-Wet" architecture can be found in [11][12]. 
The considered system applies sessile drop method [13] to calculate surface parameters. Surface tension and contact angles are calculated from images presenting melted specimens of investigated materials. Exemplary images obtained during the measurement process are presented in Figure 1. They are 8-bit monochromatic images of the resolution $320 \times 240$ pixels.

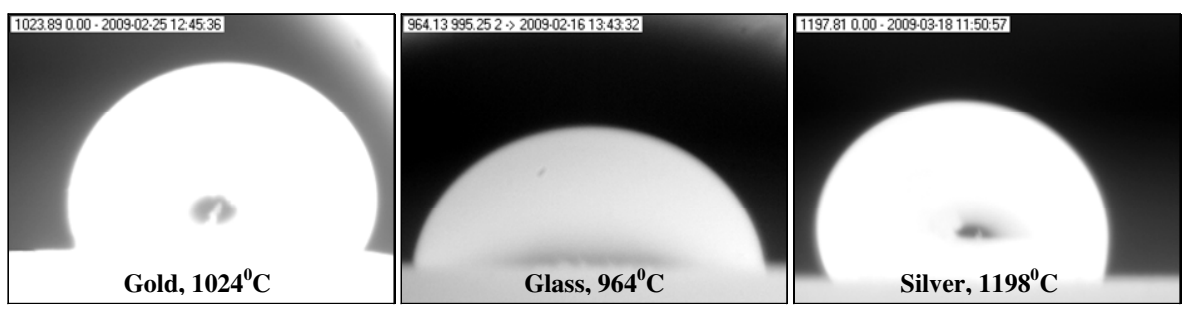

Fig. 1. Exemplary images obtained from "Thermo-Wet" vision system

After the image is acquired it is subjected to image segmentation which determines specimen shape and location of upper edge of the base-plate. Next, specimen shape analysis is carried out in order to determine characteristic geometric parameters of specimen (see Fig. 2). They are related to surface tension and contact angles through appropriate formulas arising from the sessile drop method [13]. Especially Porter's formula is applied in this stage. More detailed information about the measurement process is given in [11][12].

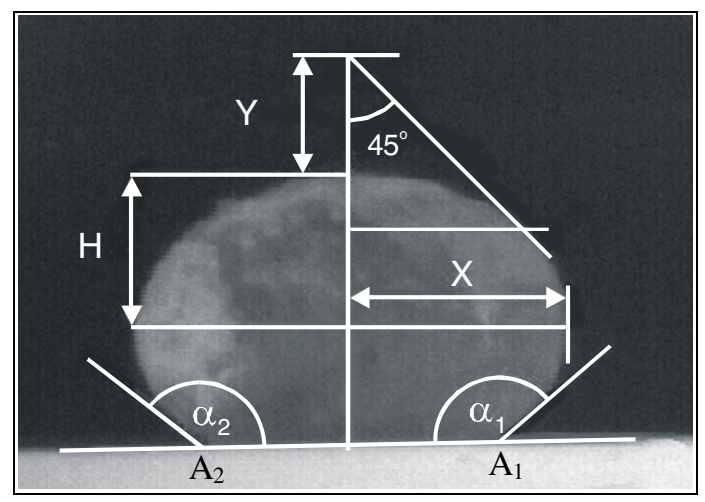

Fig. 2. The exemplary specimen with important geometric parameters marked

Image segmentation is crucial task for measurements of surface parameters. It determines specimen shape and location of upper edge of the base plate. However the segmentation of images of heat-emitting specimens is very challenging. Problems with segmentation are caused by "aura" i.e. glow that forms itself around the specimen. Aura significantly hinders accurate location of specimen edges. It blurs the border between the background and the specimen. Hard segmentation techniques join aura with the object which affect with specimen dimensions increase. 
In this paper fuzzy approach to segmentation of images presenting heat-emitting specimens is introduced. The algorithm which uses an extension of fuzzy C-means (FCM) clustering method is proposed. It iteratively segments aura. In consequence aura is effectively excluded and specimen shape is preserved after segmentation.

A brief overview of FCM and detailed description of the proposed approach are given in the following sections.

\section{Background}

Fuzzy C-means (FCM) algorithm clusters pixels into a specified number of regions (clusters). It is based on minimization of the objective function $J_{m}$ given by equation (1) $[13][14]$.

$$
J_{m}=\sum_{i \in \Omega} \sum_{j=1}^{C} u_{i j}^{m}\left\|\mathbf{x}_{i}-\mathbf{c}_{j}\right\|^{2}
$$

where:

$$
\begin{array}{rll}
m & - & \text { a real number greater than } 1 ; \\
C & - & \text { number of clusters; } \\
\mathbf{x}_{i} & - & \text { vector of pixel properties at location } i ; \\
u_{i j} & - & \text { the degree of membership of } \mathbf{x}_{i} \text { in the } j \text {-th cluster; } \\
\mathbf{c}_{j} & - & \text { centroid of the } j \text {-th cluster; } \\
\|\bullet\| & - & \text { norm expressing the distance in } P \text {-dimensional feature space; } \\
\Omega & - & \text { set of pixels in the image domain. }
\end{array}
$$

Fuzzy clustering is carried out through an iterative minimization of the objective function $J_{m}$ with the update of the degree of membership $u_{i j}$ and the cluster centers $\mathbf{c}_{j}$ by equations (2) and (3) respectively.

$$
\begin{gathered}
u_{i j}=\sum_{k=1}^{C}\left(\frac{\left\|\mathbf{x}_{i}-\mathbf{c}_{j}\right\|}{\left\|\mathbf{x}_{i}-\mathbf{c}_{k}\right\|}\right)^{-\frac{2}{m-1}} . \\
\mathbf{c}_{j}=\frac{\sum_{i \in \Omega} u_{i j}^{m} \mathbf{x}_{i}}{\sum_{i \in \Omega} u_{i j}^{m}} .
\end{gathered}
$$

The minimization is stopped when equation (4) gets fulfilled.

$$
\max _{i j}\left\{\left|u_{i j}^{(s+1)}-u_{i j}^{(s)}\right|\right\}<\varepsilon
$$

Where $\varepsilon$ is a termination criterion and $s$ is the iteration step.

After minimization of the objective function is finished maximum-membership segmentation is usually applied. During this process pixels are classified into the cluster with the highest degree of membership. 


\section{Proposed Approach}

\subsection{Pixel Description}

In case of analyzed images a pixel at location $i$ is described by a vector of features $\mathbf{x}_{i}=\left[\mathrm{x}_{i 1}, \mathrm{x}_{i 2}, \mathrm{x}_{i 3}, \mathrm{x}_{i 4}, \mathrm{x}_{i 5}, \mathrm{x}_{i 6}, \mathrm{x}_{i 7}\right]$ in 7-dimensional $(P=7)$ feature space where:

$\mathrm{x}_{i 1} \quad$ - intensity of $i$-th pixel;

$\mathrm{x}_{i 2} \quad-\quad$ an average intensity of $n \times n$ neighborhood of $i$-th pixel;

$\mathrm{x}_{i 3} \quad$ - standard deviation of intensity in $n \times n$ neighborhood of $i$-th pixel;

$\mathrm{x}_{i 4} \quad$ - gradient magnitude in $i$-th pixel;

$\mathrm{x}_{i 5} \quad-\quad$ gradient direction in $i$-th pixel;

$\mathrm{x}_{i 6} \quad-\quad$ an average gradient magnitude in $n \times n$ neighborhood of $i$-th pixel;

$\mathrm{x}_{i 7} \quad-\quad$ an average gradient direction in $n \times n$ neighborhood of $i$-th pixel.

Neighborhood of size $3 \times 3$ pixels is considered $(n=3)$. Sobel operator [1] is applied to determine magnitude and direction of gradient. The distance between pixels is computed using Euclidean metric (5).

$$
\left\|\mathbf{x}_{i}-\mathbf{x}_{j}\right\|=\sqrt{\sum_{k=1}^{P}\left(x_{i k}-x_{j k}\right)^{2}} .
$$

Extending number of features describing a pixel to proposed number of elements increases quality of image segmentation. Tests proved that using standard features i.e. pixel intensity and standard deviation is insufficient to obtain high-quality results.

\subsection{Algorithm Description}

Proposed approach clusters pixels into two $(\mathrm{C}=2)$ regions: the background $(k=1)$ and the object $(k=2)$. Clusterization is performed iteratively. In the consecutive iterations both regions compete for pixels with similar membership to both clusters.

The main steps of the algorithm are as follows:

1. Centers $\mathbf{c}_{k}^{(s)} k=\{1,2\}$ of the clusters are determined among unclassified pixels $\hat{\Omega}$.

2. Objective function $J_{m}{ }^{(s)}$ given by Equation (1) is minimized in accordance with Equations (2)-(4) for parameter $m=3$ (i.e. for each pixel $\mathbf{x}_{i}$ membership measures $u_{i k}^{(s)}\left(\mathbf{x}_{i}\right)$ in $k$ clusters are computed using fuzzy C-means clustering algorithm).

3. Pixels are temporarily assigned to clusters with maximum membership measure in accordance with equation:

$$
\forall_{i \in \hat{\Omega}} \tilde{\partial}\left(\mathbf{x}_{i}\right)=\underset{j \in[1, C]}{\arg \max }\left(u_{i j}^{(s)}\right) .
$$

where: $\tilde{\partial}\left(\mathbf{x}_{i}\right)$ is temporal affiliation of the pixel $\mathbf{x}_{i}$.

4. Among pixels temporarily assigned to $k$-th cluster threshold $T_{u k}{ }^{(s)}$ for membership in the cluster is computed. For threshold determination the ISODATA (Iterative Self-Organizing Data Analysis Technique) algorithm [14][16] is applied. 
5. Pixels $\mathbf{x}_{i}$ are permanently classified into clusters in accordance with the equation:

$$
\forall_{i \in \hat{\Omega}} u_{i k}^{(s)} \geq T_{u k}^{(s)} \Rightarrow \partial\left(\mathbf{x}_{i}\right)=k \text {. }
$$

where: $\partial\left(\mathbf{x}_{i}\right)$ is a final affiliation of the pixel $\mathbf{x}_{i}$.

In this step only pixels having the membership higher than the selected thresholds are qualified into to the regions (the object and the background). The remaining pixels are left unclassified. They are considered in the next iteration.

6. Steps $1-5$ are repeated (for $s=s+1$ ) until all pixels are classified i.e. $\hat{\Omega}=\varnothing$.

Crucial problem for the algorithm is selection of cluster centers. In the proposed approach for the first iteration the cluster centers are set manually using the knowledge about characteristic features of analyzed images. The initial cluster centers represent ideal pixel belonging to the background $\mathbf{c}_{1}{ }^{(I)}=[0,0,0,0,0,0,0]$ and ideal pixel belonging to the object $\mathbf{c}_{2}{ }^{(I)}=[255,255,0,0,0,0,0]$. In the following iterations one of the cluster centers is left unchanged and the second one is selected randomly from unclassified pixels.

For cluster centers selection bump-hunting algorithm [17] can be also used with the success. However its application increases time complexity of the proposed segmentation method.

\section{Results}

The method was extensively tested on images over a wide range of temperatures and strength of an "aura". Results of applying the proposed image segmentation algorithm to the exemplary images of heat-emitting specimens are presented in Figure 3. Moreover the figure shows a comparison with results obtained by maximum membership fuzzy C-means segmentation and the ground truths. First column presents original images. The material of the specimen and its temperature is indicated on the each image. Images with different strength of an "aura" are considered. In the second column results of image segmentation obtained using the proposed method are shown. The third column presents results of an ordinary (i.e. maximum membership) fuzzy $\mathrm{C}$-means clustering. In case of both considered segmentation methods pixels were described by vectors of features as described in Section 4.1. In the last column the ground truths are presented. They were obtained by manual segmentation performed by the skilled operator of the "Thermo-Wet" system.

Quantitative assessment of segmentation quality is presented in Table 1. Images presented in Figure 3 are considered.

Results obtained by the proposed method and maximum membership fuzzy Cmeans clustering (MM FCM) are compared with the ground truths by means of:

- good matches i.e. pixels properly qualified into both the object and the background;

- false positives i.e. background pixels qualified into the object;

- false negatives i.e. object pixels qualified into the background.

The material of the specimen and temperature describing the considered image are indicated in the column caption. 


\section{ORIGINAL IMAGE}
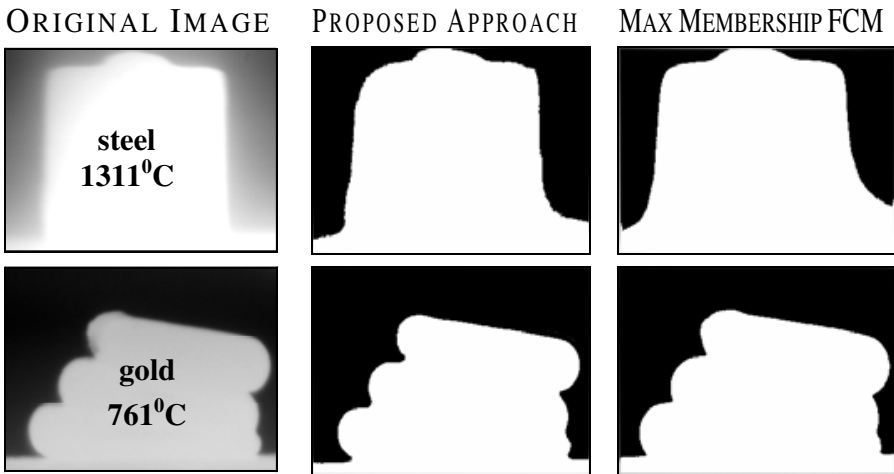

THE GROUND TRUTH
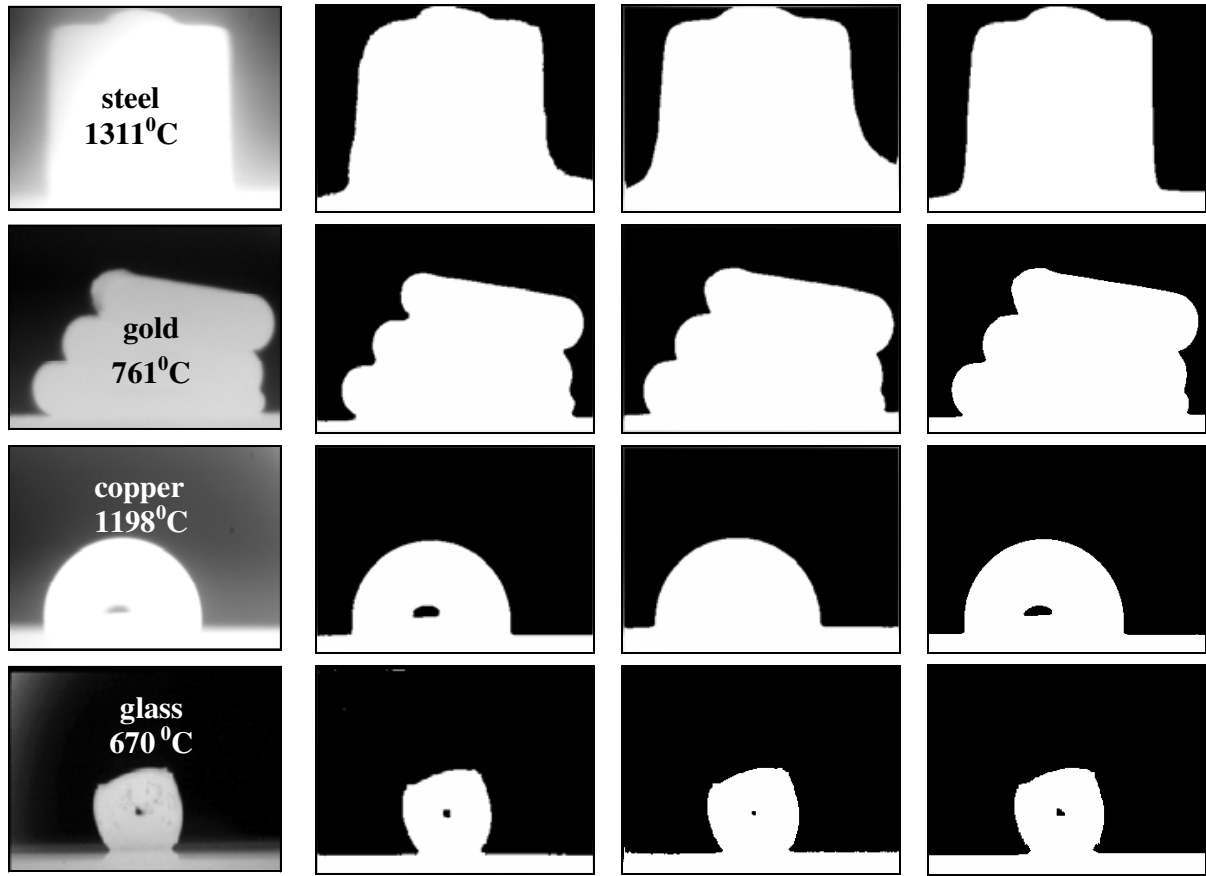

Fig. 3. Results of the proposed image segmentation algorithm compared with results obtained using an oridinary (i.e. maximum-membership) fuzzy $\mathrm{C}$-means clustering and the ground truths. The type of the image is indicated over each column.

Table 1. Quantitative assessment of image segmentation quality

\begin{tabular}{|c|c|c|c|c|c|c|c|c|}
\hline & \multicolumn{2}{|c|}{ Steel, $1311^{\circ} \mathrm{C}$} & \multicolumn{2}{|c|}{ Gold, $761^{\circ} \mathrm{C}$} & \multicolumn{2}{|c|}{ Copper, $198^{\circ} \mathrm{C}$} & \multicolumn{2}{|c|}{ Glass, $670^{\circ} \mathrm{C}$} \\
\hline & $\begin{array}{l}\text { New } \\
\text { method }\end{array}$ & $\begin{array}{l}\text { MM } \\
\text { FCM }\end{array}$ & $\begin{array}{c}\text { New } \\
\text { method }\end{array}$ & $\begin{array}{c}\text { MM } \\
\text { FCM }\end{array}$ & $\begin{array}{l}\text { New } \\
\text { method }\end{array}$ & $\begin{array}{c}\text { MM } \\
\text { FCM }\end{array}$ & $\begin{array}{l}\text { New } \\
\text { method }\end{array}$ & $\begin{array}{l}\text { MM } \\
\text { FCM }\end{array}$ \\
\hline Good matches & $96.38 \%$ & $92.86 \%$ & $98.41 \%$ & $97.10 \%$ & $99.52 \%$ & $96.81 \%$ & $99.36 \%$ & $98.97 \%$ \\
\hline False positives & $2.92 \%$ & $7.10 \%$ & $1.58 \%$ & $0.00 \%$ & $0.03 \%$ & $3.19 \%$ & $0.63 \%$ & $0.47 \%$ \\
\hline False negatives & $0.70 \%$ & $0.04 \%$ & $0.01 \%$ & $2.90 \%$ & $0.45 \%$ & $0.00 \%$ & $0.01 \%$ & $0.56 \%$ \\
\hline
\end{tabular}

\section{Discussion}

Results presented in Figure 3 and Table 1 show clearly that the proposed extension of a fuzzy C-means segmentation algorithm efficiently segments images of heat-emitting specimens. High quality results are obtained for images of different intensity and strength of an "aura". Shape of objects after segmentation is well defined. Obtained contours are smooth, regular and free from defects. Moreover, important details of specimen shape are properly extracted.

Both the visual and the quantitative comparison with results obtained by maximum-membership fuzzy C-means segmentation prove that the proposed approach is much more accurate in segmenting an "aura". While traditional FCM joins aura with 
the object and significantly increases its dimensions, the new algorithm preserves specimen dimensions by qualifying an "aura" to the background. The difference between results obtained by both considered methods can especially be observed in case of images with significant aura.

Tests proved that in case of analyzed class of images the proposed segmentation algorithm finishes in less than ten iterations.

\section{Conclusions}

In this paper problem of fuzzy image segmentation was considered. Especially an extension of fuzzy C-means segmentation algorithm was introduced. The algorithm does not perform crisp classification of pixels into clusters with maximum membership measure but makes background and object compete for pixels in consecutive iterations.

Presented results prove that the proposed algorithm performs accurate segmentation of images of heat-emitting specimens. Specimen shape after segmentation is well defined - much better than in case of an ordinary FCM clusterization. Segmented objects are characterized by smooth and regular borders. Moreover an "aura" (i.e. glow that forms itself around the specimen) is effectively removed by the new approach. Traditional FCM approach joins aura with the object what increases object dimensions. Quality of obtained results is sufficient for further quantitative analysis of specimen shape.

Although the algorithm has been developed for a certain class of images, it can be successfully applied in a wide spectrum of applications as it does not take the advantage of knowledge about image properties.

\section{Acknowledgements}

The author is a scholarship holder of project entitled "Innovative education ..." supported by European Social Fund.

\section{References}

1. Gonzalez, R., Woods, E.: Image Processing. Prentice Hall, New Jersey (2007)

2. Liu, X., Wang, D.: Image and Texture Segmentation Using Local Spectral Histograms. IEEE Trans. Image Proc. 15(10), 3066-3077 (2006)

3. Fan, J., Zengb, G., Bodyc, M., Hacidc, M.: Seeded region growing: an extensive and comparative study. Pattern Recognition Letters 26(8), 1139-1156 (2005)

4. Silva, L., Bellon, O., Gotardo, P.: Edge-based image segmentation using curvature sign maps from reflectance and range images. In: IEEE Int. Conf. Image Processing, vol. 1, pp. 730-733 (2001)

5. Bo, S., Ma, Y., Zhu, C.: Image Segmentation by Nonparametric Color Clustering. In: Shi, Y., van Albada, G.D., Dongarra, J., Sloot, P.M.A. (eds.) ICCS 2007. LNCS, vol. 4489, pp. 898-901. Springer, Heidelberg (2007) 
6. Haris, K., Efstratiadis, S., Maglaveras, N., Katsaggelos, A.: Hybrid image segmentation using watersheds and fast region merging. IEEE Trans. Image Proc. 7(12), 1684-1699 (1998)

7. Zadeh, L.: Fuzzy sets. Information and Control 8(3), 338-353 (1965)

8. Zadeh, L.: Fuzzy logic = computing with words. IEEE Trans. Fuzzy Systems 4(2), 103 111 (1996)

9. Tizhoosh, H.: Fuzzy ImageProcessing. Introduction in Theory and Practice. Springer, Berlin (1997)

10. Chi, Z., Yan, H., Pahm, T.: Fuzzy Algorithms: With Applications to Image Processing and Pattern Recognition. In: Advances in Fuzzy Systems. Applications and Theory, 10. World Scientific Pub. Co. Inc (1996)

11. Sankowski, D., Strzecha, K., Jeżewski, S.: Digital image analysis in measurement of surface tension and wet ability angle. In: Int. Conf. Modern Problems of Telecommunications, Computer Science and Engineers Training, Lviv-Slavskie, Ukraine, pp. 129-130 (2000)

12. Sankowski, D., Senkara, J., Strzecha, K., Jeżewski, S.: Automatic investigation of surface phenomena in high temperature solid and liquid contacts. In: IEEE Instrumentation and Measurement Technology Conference, Budapest, Hungary, pp. 1397-1400 (2001)

13. Adamson, A., Gast, A.: Physical Chemistry of Surfaces. Wiley-Interscience, USA (1997)

14. Dunn, J.: A Fuzzy Relative of the ISODATA Process and its Use in Detecting Compact, Well Separated Clusters. J. Cyber. 3, 32-57 (1974)

15. Bezdek, J.: Pattern Recognition with Fuzzy Objective Function Algorithms. Plenum, New York (1981)

16. Ridler, T., Calvard, S.: Picture thresholding using an iterative selection method. IEEE Trans. Syst. Man Cyb. 8, 630-632 (1978)

17. Silverman, B.: Density Estimation for Statistics and Data Analysis. Chapman \& Hall, London (1993) 